\title{
Quadruple Therapy Offers High SVR Rates in Patients with HCV Genotype 4 with Previous Treatment Failure
}

\author{
Yousry Esam-Eldin Abo-amer, ${ }^{1}$ Rehab Badawi, ${ }^{2}$ Mohamed El-Abgeegy, ${ }^{3}$ \\ Heba Fadl Elsergany, ${ }^{3}$ Ahmed Abdelhaleem Mohamed, ${ }^{3}$ Sahar Mohamed Mostafa, ${ }^{3}$ \\ Hatem Samir Alegaily, ${ }^{4}$ Shaimaa Soliman, ${ }^{5}$ Sally Elnawasany, ${ }^{2}$ and Sherief Abd-Elsalam $\mathbb{D}^{2}$ \\ ${ }^{1}$ Hepatology, Gastroenterology, and Infectious Diseases Department, Mahala Hepatology Teaching Hospital, Gharbia, Egypt \\ ${ }^{2}$ Tropical Medicine Department, Tanta University, Tanta, Egypt \\ ${ }^{3}$ Hepatology and Liver Transplantation Departments, National Hepatology and Tropical Medicine Research Institute, \\ Cairo, Egypt \\ ${ }^{4}$ Hepatology, Gastroenterology, and Infectious Diseases Department, Benha Faculty of Medicine, Benha, Egypt \\ ${ }^{5}$ Department of Public Health and Community Medicine, Menofia University, Menofia, Egypt \\ Correspondence should be addressed to Sherief Abd-Elsalam; sherif_tropical@yahoo.com
}

Received 7 February 2020; Revised 11 July 2020; Accepted 13 July 2020; Published 24 July 2020

Academic Editor: Marco Ciotti

Copyright (c) 2020 Yousry Esam-Eldin Abo-amer et al. This is an open access article distributed under the Creative Commons Attribution License, which permits unrestricted use, distribution, and reproduction in any medium, provided the original work is properly cited.

\begin{abstract}
Background and Aims. Direct-acting antivirals (DAAs) have made a revolution in hepatitis C virus (HCV) treatment with promising reduction of $\mathrm{HCV}$ infection and disease morbidities. However, unfortunately, treatment failure still occurs in about $5-15 \%$ of patients treated with DAA-based combination regimens. The primary aim of the study was to assess the efficacy and safety of a quadruple regimen of (sofosbuvir, daclatasvir, and simeprevir with a weight-based ribavirin) in chronic HCV DAAsexperienced patients. Methods. This observational, open-label prospective study was carried out on 103 genotype 4 hepatitis C virus-infected patients who failed to achieve SVR12 after sofosbuvir-daclatasvir with or without ribavirin. Patients were treated for three months with sofosbuvir $(400 \mathrm{mg})$, daclatasvir $(60 \mathrm{mg})$, and simeprevir $(150 \mathrm{mg})$ with a weight-based ribavirin dosage $(1000-1200 \mathrm{mg} / \mathrm{d})$. Response to treatment was determined by quantitative PCR for HCV at 3 months after the end of treatment (SVR12), and adverse events during the treatment were recorded. Results. SVR was achieved in 100 patients (97.1\%) at week 12 after treatment. No dangerous or life-threatening adverse events were recorded. Conclusions. Retreatment of HCV genotype 4 patients with quadruple therapy is a good therapeutic option and achieves high response rates with minimal side effects.
\end{abstract}

\section{Introduction}

Chronic Hepatitis C infection (HCV) constitutes a worldwide health problem. In 2015, the world health organization (WHO) reported 71 million $\mathrm{HCV}$ chronically infected people and 1.34 million of chronic liver disease and primary liver cancer-related deaths $[1,2]$.

Direct-acting antivirals (DAAs) have made a revolution in $\mathrm{HCV}$ treatment with promising reduction of $\mathrm{HCV}$ infection and disease morbidities [3-8] International liver societies proved DAA-based combination regimens as a new standard of care treatment for chronic $\operatorname{HCV}[9,10]$.
Treatment with at least two DAAs for hepatitis $\mathrm{C}$ virus (HCV) genotype 1 or 4 is associated with more than $90 \%$ sustained virological response (SVR) rates [11-15].

Chronic hepatitis C patients were treated in 2016 with a combination of 1 to 3 DAAs of 4 groups, with or without ribavirin $[16,17]$. It is noteworthy mentioning that NS5B inhibitors have a high resistance barrier because the variables you choose modestly reduce the susceptibility to these drugs and lower fitness. Hence, penetration or relapse is the exception when these medications are given as a monotherapy. On the other hand, NS5A inhibitors and protease inhibitors NS3-4A have low barriers to resistance. When 
given as monotherapy, they quickly select the appropriate resistance variants. Second-generation NS3-4A and NS5A inhibitors have increased barriers to resistance. They are substantially more active against many but not all variants resistant to first-generation compounds [18].

Unfortunately, treatment failure still occurs in about $5-15 \%$ of patients treated with DAA-based combination regimens. Treatment failure of $\mathrm{HCV}$ may be the result of viral factors (HCV genotype and the presence of resistant variants), host factors (fibrosis and portal hypertension), and treatment-related factors (patient's adherence, duration of therapy metabolism of the drugs, and ribavirin use) [19-22].

These viral factors that lead to failure are attributed to the selection existence of $\mathrm{HCV}$ viral variants that resist the used DAA [23-26].

Retreatment after DAA failure is a challenge, especially in those for whom NS5A inhibitors-based regimens with cross-resistance across all members of the drug class [25-27]. Together with the wide spread use of NS5A inhibitors-based regimens, this leads to long-term persistence of resistant variants that convey viral resistance up to 96 weeks after treatment failure [23, 28, 29].

The current updates of the international HCV treatment guidelines recommend a single-tablet combination of SOF plus velpatasvir and voxilaprevir (SOF/VEL/VOX) for 12 weeks as the standard treatment after failure of NS5A-based regimens. Addition of $\mathrm{RBV}$ or extension of the treatment duration of the SOF/VEL/VOX regimen, as well as combining SOF with glecaprevir and pibrentasvir (GLE/PIB), could be considered in difficult-to-treat patients [10].

On the other hand, NS3/4A protease inhibitors induce a shorter term of resistance [30], and when added to a highresistance barrier of sofosbuvir, as well as, to protease inhibitors (that lack cross-resistance between NS5A inhibitors), they present a reasonable option for retreatment of NS5A-containing regimen failures [22, 26-34].

It is worth mentioning that due to the limited availability of some newer DAAs in Egypt, the national committee for viral hepatitis management recommended treatment of hepatitis $\mathrm{C}$ patients for whom previous SOF/DCV-based regimens have failed with a combination of either SOF plus ritonavir boosted with paritaprevir and ombitasvir (OBV/ $\mathrm{PTV} / \mathrm{r}) \pm \mathrm{RBV}$ or SOF plus simeprevir (SMV) and $\mathrm{DCV} \pm \mathrm{RBV}$ for 12 or 24 weeks according to RBV eligibility [19].

We aimed in this study to evaluate the efficacy and safety of combining sofosbuvir, daclatasvir, and simeprevir with ribavirin in chronic HCV DAAs-experienced patients.

\section{Methods}

This observational, open-label prospective study was carried out on 103 genotype 4 hepatitis C virus-infected patients who failed to achieve SVR12 after sofosbuvir and daclatasvir with or without ribavirin.

All patients were attending to an insurance hospital clinic and outpatient clinics of a major University hospital and a major research institute during the period of April 2018 to October 2018. All patients had positive quantitive
PCR for HCV infection after failure of sustained virological response at 12 weeks after the end of treatment. Institutional ethical committee approval was obtained before the start of the study, and a written consent was taken from every participant. The study protocol conforms to the ethical guidelines of the 1975 Declaration of Helsinki as reflected in a prior approval by the institution's human research committee.

The study enrolled all the patients who agreed to participate and who were within the inclusion criteria of the study. Inclusion criteria were patients aged 18 years or older who are treatment-experienced compensated Child-Pugh A cirrhotic patients and were HCV positive RNA by PCR at 12 weeks after the end of treatment with sofosbuvir and daclatasvir with or without ribavirin.

The exclusion criteria were (a) decompensated cirrhosis, (b) organ transplantation, (c) severe uncontrolled morbidity, e.g., cardiac, (e) malignant Tumors, (f) HIV or hepatitis B virus coinfection, (g) pregnancy or lactation, and (h) receiving contraindicated concomitant drugs for sofosbuvir, daclatasvir, and simeprevir plus ribavirin.

All patients enrolled in this study were evaluated by the complete blood count (CBC), liver function tests, fasting blood sugar (FBS), serum creatinine, HBs-Ag testing, and $\alpha$-fetoprotein (AFP), as well as abdominal ultrasonography.

Patients were treated for 12 weeks with a combination of sofosbuvir (SOF) (400 mg), daclatasvir (DAC) $(60 \mathrm{mg})$, and simeprevir (SIM) (150 mg) with a weight-based ribavirin dosage RBV (1000-1200 mg/d).

Follow-up of the patients was routinely conducted every 4 weeks when the patients attended the clinic to receive their prescriptions. Any side effects noticed or complained by the patients were recorded. If there were side effects, additional visits were recommended.

Complete blood count, liver and kidney function tests, and PCR for HCV were performed before the start of therapy and at weeks 4,8 , and 12 during antiviral treatment.

End of treatment response was determined by quantitative PCR for HCV at end of treatment for each of the study groups.

The primary outcome measures were the number of patients with successful virus eradication at 12 weeks after discontinuation of therapy (SVR12). SVR was defined as negative HCV-RNA 12 weeks after completion of DAA therapy.

The secondary end point was to evaluate the side effects and the safety of this regimen in our patient groups.

2.1. Statistical Analysis. Data were expressed in Number (No), percentage $(\%)$, mean $(\bar{x})$, and standard deviation (SD). Kolmogorov-Smirnov and Shapiro-Wilk tests were used to test the normality of different variables. Repeated measures ANOVA (with the Bonferoni correction) with the Mauchly test as a sphereicty test were used for comparison among three or more consecutive measures in the same group of quantitative variables. Assumed spherecity was used for normally distributed data, while Greenhouse-Geisser was used for not normally distributed data. A 
two-sided $p$ value of $<0.05$ was considered statistically significant. Statistical Package for Social Science version 23 was used for performing the statistical analysis (SPSS Inc. Released 2015. IBM SPSS statistics for windows, version 23.0, Armnok, NY:IBM Corp.).

\section{Results}

A total of 103 patients with HCV GT-4 infection who failed to achieve SVR12 after SOF $+\mathrm{DAC} \pm \mathrm{RBV}$ were treated per protocol with SIM + SOF + DAC + RBV for 12 weeks. The mean age was $54.48 \pm 4.81$ years, and $56(54.4 \%)$ patients were male. Baseline demographic and clinical characteristics are shown in (Table 1).

There were no significant changes in the level of followup of white blood cells ( $p$ value $=0.625)$, platelet ( $p$ val$\mathrm{ue}=0.580)$, serum creatinine $(p$ value $=0.43)$, and total bilirubin when they were compared to the pretreatment level or 12 weeks after treatment. Pretreatment hemoglobin was significantly higher than week $4(0.008)$ and, then, in all other weeks $(p<0.001)$; also, it was significantly higher at week 4 when compared to its level at week 8 and week 12 $(<0.001)$.

Hemoglobin was significantly higher at week 12 after treatment than its level at week 8 and and week $12(<0.001)$. Pretreatment levels of ALT and AST were significantly higher than in all weeks of treatment $(p<0.001)$ (Table 2).

Assessment of the effectiveness of antiviral treatment: end of treatment response was achieved in all 103 patients $(100 \%)$ at week 12, while SVR was achieved in 100 patients (97.1\%) at week 12 after treatment (Table 3).

The safety of antiviral treatment: fifty patients (47.5\%) had side effects in the form of anemia in 30 patients (29.1\%), pruritus in 43 patients (41.7\%), headache in 5 patients (4.9\%), fatigue in 22 patients (21.4\%), insomnia in 7 patients (7.8\%), diarrhea in 6 patients $(5.8 \%)$, nausea in 3 patients (2.9\%), cough in 6 patients (8.7\%), and myalgia in 2 patients (1.9\%) (Table 4).

\section{Discussion}

Our goal in this study was to assess the efficacy, safety, and tolerability of sofosbuvir, daclatasvir, simeprevir, and ribavirin in 12 weeks treatment in direct-acting antiviral-experienced patients. One hundred and three genotype 4compensated Child-Pugh A cirrhotic patients were enrolled in the study.

Regarding biochemical parameters, basal hemoglobin was significantly higher than its levels in week $4(0.008)$ and all other weeks $(p<0.001)$. Moreover, the hemoglobin level at week 4 showed significant increment than its levels at week 8 and week $12(<0.001)$. At week 12 after treatment, hemoglobin significantly increased than at w 8 and $\mathrm{w} 12$ $(<0.001)$, which was mainly attributed to the ribavirin effect. Baseline levels of ALT and AST were significantly higher than at all weeks of therapy $(p<0.001)$.

End of treatment response was achieved in all 103 patients (100\%), while SVR12 was achieved in 100 patients $(97.1 \%)$ at week 12 after treatment.
TABle 1: Baseline demographic and clinical characteristics $(n=103)$.

\begin{tabular}{lc}
\hline Variable & Pretreatment mean \pm SD, range \\
\hline Age & $54.48 \pm 4.81,27.0-71.0$ \\
Sex & $56(54.4)$ \\
Male & $47(45.6)$ \\
Female & $34(33.0)$ \\
Hypertension & $31(30.01)$ \\
DM & $103.72 \pm 43.32,71.0-299.0$ \\
FBG & $7.62 \pm 0.66,6.60-8.10$ \\
HbAlc $(n=27)$ & $13.87 \pm 38.57,0.70-276.0$ \\
AFP & $0(0.0)$ \\
HBs-Ag & $103(100.0)$ \\
Positive & $80.66 \pm 10.54,55.0-100.0$ \\
Negative & $3.79 \pm 0.47,3.0-4.80$ \\
Prothrombin activity & $414722.16 \pm 766382.13$, \\
Serum albumin & $654.0-4002092.0$ \\
Viral load & $100(100.0)$ \\
End of treatment & \\
response & $3(3.0)$ \\
Sustained viral response & $97(97.0)$ \\
Positive & \\
Negative & $8(8.0)$ \\
Ribavirin modification & $92(92.0)$ \\
Positive & $58(58.0)$ \\
Negative & $42(42.0)$ \\
Previous treatment &
\end{tabular}

Only the IMPACT study evaluated a ribavirin-free version of the same regimen administered for 12 weeks in 40 treatment-naive or treatment-experienced patients with decompensated cirrhosis or portal hypertension. There was discontinuation of treatment due to adverse events occurred, and a $100 \%$ SVR12 rate was achieved. This difference can probably be explained by the fact that our patients had already experienced a failed DAA-based regimen [35].

At the level of real world studies, our findings were similar in regimen but higher in SVR to the results of Hézode et al. [32], where they evaluated the effect of sofosbuvir, daclatasvir, simeprevir, and ribavirin 24 -week regimen in 10 direct-acting antiviral-experienced patients. The study included genotypes $1,2,4$, and 6 . Relapse occurred in 2 patients, and they were of genotype 1 .

The inclusion of many HCV genotypes in the study of Hézode et al. together with the small number of their patients may explain the difference between the SVR 12 percentages in our study [32].

Our results was quite similar to those of a recently published Egyptian study of Said et al. 2020 who used the same regimen and recorded 96\% of SVR12 [36]

Similar studies, but without the same drug regimen, were conducted. In 2015, Lawitz et al. carried out a ribavirin-free regimen (SOF, DAC, and SIM) for 12 weeks in 40 treatmentnaive or treatment-experienced patients with decompensated cirrhosis or portal hypertension. There were no side effects, and SVR12 was 100\% [35]. 
TABLE 2: Baseline and follow-up laboratory investigations of the studied group $(n=103)$.

\begin{tabular}{|c|c|c|c|c|c|c|}
\hline Variable: & Pretreatment mean \pm SD & $\mathrm{W} 4$ mean $\pm \mathrm{SD}$ & $\mathrm{W} 8$ mean $\pm \mathrm{SD}$ & W12 & W12 post & $p$ value \\
\hline Serum creatinine & $0.89 \pm 0.25$ & $0.89 \pm 0.23$ & $0.93 \pm 0.26$ & $0.90 \pm 0.14$ & $0.90 \pm 0.11$ & 0.43 \\
\hline WBCs & $6613.0 \pm 2298.8$ & $6756.00 \pm 2244.51$ & $6721.0 \pm 2046.3$ & $6626.5 \pm 1909.9$ & $6632.0 \pm 1857.3$ & 0.625 \\
\hline $\mathrm{HB}$ & $13.65 \pm 1.70$ & $13.11 \pm 1.59$ & $12.16 \pm 1.76$ & $12.12 \pm 1.88$ & $12.90 \pm 1.18$ & $<0.001$ \\
\hline Plt & $152.51 \pm 47.69$ & $160.78 \pm 65.09$ & $154.10 \pm 56.17$ & $163.07 \pm 55.88$ & $161.25 \pm 52.04$ & 0.580 \\
\hline ALT & $53.39 \pm 35.28$ & $27.71 \pm 13.28$ & $24.77 \pm 10.60$ & $24.85 \pm 6.87$ & $24.59 \pm 5.89$ & $<0.001$ \\
\hline AST & $51.55 \pm 37.35$ & $28.83 \pm 14.28$ & $25.24 \pm 9.36$ & $26.07 \pm 6.18$ & $25.82 \pm 5.38$ & $<0.001$ \\
\hline Total bilirubin & $1.10 \pm 0.45$ & $1.24 \pm 0.70$ & $1.16 \pm 0.61$ & $1.21 \pm 0.51$ & $1.13 \pm 0.37$ & 0.104 \\
\hline
\end{tabular}

TABLE 3: Virological response of studied patients.

\begin{tabular}{lcc}
\hline Time & SVR & 95\% CI \\
\hline End of treatment response & $103 / 103(100.0 \%)$ & $100.0-100.0$ \\
Sustained virological response (12 weeks after treatment) & $100 / 103(97.1 \%)$ & $93.2-100.0$ \\
\hline
\end{tabular}

TABLE 4: Side effects in the studied patients.

\begin{tabular}{lc}
\hline Side effects & No. $(\%)(n=103)$ \\
\hline${ }^{*}$ No side effects & $53(51.5)$ \\
${ }^{*}$ With side effects & $50(47.5)$ \\
Anemia & \\
Pruritus & $30(29.1)$ \\
Headache & $43(41.7)$ \\
Fatigue & $5(4.9)$ \\
Insomnia & $22(21.4)$ \\
Diarrhea & $7(7.8)$ \\
Nausea & $6(5.8)$ \\
Cough & $3(2.9)$ \\
Myalgia & $6(8.7)$ \\
\end{tabular}

Another study aimed to test the efficacy of 12 weeks treatment with SOF plus SIM, without RBV in NS5Aexperienced patients. They enrolled 16 patients (genotype 1: $14 / 16$ and genotype $4: 2 / 16$ ) with advanced fibrosis or compensated cirrhosis. SVR12 was 87.5; the two patients who relapsed were infected with HCV GT 1a, cirrhotic, had relatively high HCV-RNA levels at baseline, and had at least one RAV detected in both the NS3 and NS5A regions [37].

On the same track; Wahsh et al. [38] evaluated the safety and efficacy of simeprevir plus sofosbuvir in 12 week treatment of HCV-naïve and -experienced 175 cirrhotic and noncirrhotic patients, where the liver enzymes showed significant decline at the end of therapy, and SVR12 was achieved in $97.7 \%$ of cirrhotic patients who showed lower SVR (92.7\%). Mild and tolerable adverse effects were detected in $57.14 \%$ of patients mainly in the form of headache, fatigue, pruritus, dizziness, and photosensitivity [38, 39].

As a comparison between our findings and the FDAapproved VOSEVI regimen which is a combination of three antiviral drugs, sofosbuvir (SOF) and velpatasvir (VEL) and voxilaprevir (VOX), this combination was approved to be used in treatment-experienced chronic HCV patients without cirrhosis or with compensated cirrhosis, on the basis of two clinical trials of 748 chronic HCV patients [40].
In our study, the SVR rate was also comparable to those reported by Bourlière et al. in the POLARIS-1study [40], in which the triple combination of SOF/VEL/VOX was used for 12 weeks to treat $\mathrm{CHC}$ patients after an unsuccessful NS5A-containing DAA regimen. The overall SVR rate was $96 \%(253 / 263)$ of treated patients, while the SVR was $91 \%$ in patients with HCV genotype $4(20 / 22$ patients) $[40,41]$.

Regarding the safety of the used antiviral regimen, fifty patients $(47.5 \%)$ had side effects in the form of anemia in 30 patients $(29.1 \%)$, pruritus in 43 patients $(41.7 \%)$ headache in 5 patients $(4.9 \%)$, fatigue in 22 patients $(21.4 \%)$, insomnia in 7 patients $(7.8 \%)$, diarrhea in 6 patients $(5.8 \%)$, nausea in 3 patients $(2.9 \%)$, cough in 6 patients $(8.7 \%)$, and myalgia in 2 patients $(1.9 \%)$.

The relative high percentage of anemia is attributed to ribavirin in the used regimen and to cirrhosis of the study population.

Simeprevir is known to induce many skin adverse effects, the commonest of which is severe itching that explains the obvious pruritus rate in our patients [39].

On the other hand, Hézode et al. [32] reported that 2 patients met severe side effects such as pulmonary arterial hypertension and acute-on-chronic liver failure which may be attributed to the presence of cirrhosis and NS5A and/or NS3 protease resistance-associated [22, 33, 34].

The main limitation of the study is the limited sample size. So, more studies on the broader number of patients with genotype 4 are required to document the safety and efficacy of this regimen on this special category of patients.

In conclusion, the combination of sofosbuvir ( $400 \mathrm{mg}$ ), daclatasvir $(60 \mathrm{mg})$, and simeprevir $(150 \mathrm{mg})$ with a weightbased ribavirin dosage $(1000-1200 \mathrm{mg} / \mathrm{d})$ is effective with moderate tolerable side effects in retreatment of compensated cirrhotic patients who failed to respond to previous DAA-containing regimens.

\section{Data Availability}

The authors' institution does not allow public data access. 


\section{Ethical Approval}

This research was approved by the Tanta University Faculty of Medicine Research ethical Committee. The study protocol complies with the ethical guidelines of the 1975 Declaration of Helsinki as reflected in prior approval by the institution's Human Research Committee.

\section{Consent}

Written informed consent was obtained from each patient.

\section{Conflicts of Interest}

The authors declare that there are no conflicts of interest.

\section{Authors' Contributions}

All the authors participated sufficiently in the work and approved the final version of the manuscript.

\section{Acknowledgments}

The authors acknowledge all patients participated in this study and take responsibility for all aspects of the reliability and freedom from bias of the data presented and their discussed interpretation.

\section{References}

[1] World Health Organization (WHO), Gloal Hepatitis Report, World Health Organization (WHO), Geneva,Switzerland, 2017, https:/www.who.int/hepatitis/publications/global-hepatitisreport2017/en/.

[2] O. A. Ahmed, E. Safwat, M. O. Khalifa et al., "Sofosbuvir plus daclatasvir in treatment of chronic hepatitis $C$ genotype 4 infection in a cohort of Egyptian patients: an experiment the size of Egyptian village," International Journal of Hepatology, vol. 2018, Article ID 9616234, 5 pages, 2018.

[3] R. S. Brown and P. J. Gaglio, "Scope of worldwide hepatitis C problem," Liver Transplantation, vol. 9, no. 11, pp. 10-13, 2003.

[4] H. H. Youb and L. J. Abu-Raddad, "Impact of treatment on hepatitis $\mathrm{C}$ virustransmission and incidence in Egypt: a case for treatment as prevention," Journal of Viral Hepatitis, vol. 24, no. 6, pp. 486-495, 2017.

[5] O. A. Ahmed, H. H. Kaisar, N. Hawash et al., "Efficacy of sofosbuvir plus ribavirin with or without peginterferon-alfa in treatment of a cohort of Egyptian patients with hepatitis C virus infection," Infectious Disorders-Drug Targets, vol. 17, no. 2, pp. 95-100, 2017.

[6] S. Abd-Elsalam, M. Sharaf-Eldin, S. Soliman, A. Elfert, R. Badawi, and Y. K. Ahmad, "Efficacy and safety of sofosbuvir plus ribavirin for treatment of cirrhotic patients with genotype 4 hepatitis C virus in real-life clinical practice," Archives of Virology, vol. 163, no. 1, pp. 51-66, 2018.

[7] O. A. Ahmed, H. H. Kaisar, R. Badawi et al., "Efficacy and safety of sofosbuvir-ledipasvir for treatment of a cohort of Egyptian patients with chronic hepatitis C genotype 4 infection," Infection and Drug Resistance, vol. 11, pp. 295-298, 2018.

[8] O. A. Ahmed, M. A. Elsebaey, M. H. A. Fouad et al., "Outcomes and predictors of treatment response with sofosbuvir plus daclatasvir with or without ribavirin in Egyptian patients with genotype 4 hepatitis C virus infection," Infection and Drug Resistance, vol. 11, pp. 441-445, 2018.

[9] J.-M. Pawlotsky, F. Negro, A. Aghemo et al., "EASL recommendations on treatment of hepatitis C 2018," Journal of Hepatology, vol. 69, no. 2, pp. 153-194, 2018.

[10] AASLD-IDSA, Recommendations for Testing, Managing, and Treating Hepatitis C, 2018, http://www.hcvguidelines.org.

[11] M. Buti, M. Riveiro-Barciela, and R. Esteban, "Management of direct-acting antiviral agent failures," Journal of Hepatology, vol. 63, no. 6, pp. 1511-1522, 2015.

[12] D. Dieterich, B. Bacon, S. Flamm et al., "Final evaluation of $955 \mathrm{HCV}$ patients treated with 12 week regimens containing sofosbuvir $+/-$ simeprevir in the TRIO network: academic and community treatment of a real-world, heterogeneous population," Journal of Hepatology, vol. 62, p. S621, 2015.

[13] D. M. Jensen, J. G. O'Leary, P. J. Pockros et al., "Safety and efficacy of sofosbuvir-containing regimens for hepatitis C: real-world experience in a diverse, longitudinal observational cohort," Hepatology, vol. 60, p. S219, 2014.

[14] S. Pol, M. Bourliere, S. Lucier et al., "Safety and efficacy of daclatasvir-sofosbuvir in HCV genotype 1-mono-infected patients," Journal of Hepatology, vol. 66, pp. 39-47, 2017.

[15] M. S. Sulkowski, H. E. Vargas, A. M. Di Bisceglie et al., "Effectiveness of simeprevir plus sofosbuvir, with or without ribavirin, in real-world patients with HCV genotype 1 infection," Gastroenterology, vol. 150, pp. 419-429, 2016.

[16] J. M. Pawlotsky, "New hepatitis C therapies: the toolbox, strategies, and challenges," Gastroenterology, vol. 146, pp. 1176-1192, 2014.

[17] J.-M. Pawlotsky, "Hepatitis C treatment: the data flood goes on. An update from the liver meeting 2014," Gastroenterology, vol. 148, no. 3, pp. 468-479, 2015.

[18] J.-M. Pawlotsky, "Hepatitis C virus resistance to direct-acting antiviral drugs in interferon-free regimens," Gastroenterology, vol. 151, no. 1, pp. 70-86, 2016.

[19] A. S. Hanafy, S. Soliman, and S. Abd-Elsalam, "Rescue therapy for chronic hepatitis $\mathrm{C}$ virus infection after repeated treatment failures: impact on disease progression and risk of hepatocellular carcinoma," Hepatology Research, vol. 49, no. 4, pp. 377-384, 2019.

[20] G. Abdelmoemen, S. A. Khodeir, S. Abou-Saif, A. Kobtan, and S. Abd-Elsalam, "Prevalence of occult hepatitis $\mathrm{C}$ virus among hemodialysis patients in Tanta university hospitals: a singlecenter study," Environmental Science and Pollution Research, vol. 25, no. 6, pp. 5459-5464, 2018.

[21] V. Costilla, N. Mathur, and J. A. Gutierrez, "Mechanisms of virologic failure with direct-acting antivirals in hepatitis $\mathrm{C}$ and strategies for retreatment," Clinics in Liver Disease, vol. 19, no. 4, pp. 641-656, 2015.

[22] L. N. Cavalcante and A. C. Lyra, "Predictive factors associated with hepatitis C antiviral therapy response," World Journal of Hepatology, vol. 7, no. 12, pp. 1617-1631, 2015.

[23] European Association for the Study of the Liver, "EASL recommendations on treatment of hepatitis C 2015," Journal of Hepatology, vol. 63, no. 1, pp. 199-236, 2015.

[24] E. Lawitz, S. Flamm, J. C. Yang et al., "Retreatment of patients who failed 8 or 12 weeks of ledipasvir/sofosbuvir-based regimens with ledipasvir/sofosbuvir for 24 weeks," Journal of Hepatology, vol. 62, no. 2, p. S192, 2015.

[25] E. Poveda, D. L. Wyles, A. Mena et al., "Update on hepatitis C virus resistance to direct-acting antiviral agents," Antiviral Research, vol. 108, pp. 181-191, 2014. 
[26] J. M. Pawlotsky, "Treatment failure and resistance with directacting antiviral drugs against hepatitis C virus," Hepatology, vol. 53, pp. 1742-1751, 2011.

[27] M. Gao, "Antiviral activity and resistance of HCV NS5A replication complex inhibitors," Current Opinion in Virology, vol. 3, no. 5, pp. 1-7, 2013.

[28] F. McPhee, D. Hernandez, F. Yu et al., "Resistance analysis of hepatitis $\mathrm{C}$ virus genotype 1 prior treatment null responders receiving daclatasvir and asunaprevir," Hepatology, vol. 58, no. 3, pp. 902-911, 2013.

[29] D. L. Wyles, P. Ruane, M. S. Sulkowski et al., "Daclatasvir plus sofosbuvir for treatment of HCV genotypes 1-4 in HIV-HCV coinfection: the ALLY-2 study," J Hepatol, vol. 62, no. 2, p. S263, 2015.

[30] O. Lenz, T. Verbinnen, B. Fevery et al., "Virology analyses of $\mathrm{HCV}$ isolates from genotype 1-infected patients treated with simeprevir plus peginterferon/ribavirin in phase IIb/III studies," Journal of Hepatology, vol. 62, no. 5, pp. 1008-1014, 2015.

[31] C. Sarrazin, H. Dvory-Sobol, E. Svarovskaia et al., "Baseline and post-baseline resistance analyses of Phase $2 / 3$ studies of ledipasvir/sofosbuvir \pm RBV," Hepatology, vol. 60, no. 1 , p. 1128A, 2014.

[32] C. Hézode, S. Fourati, S. Chevaliez et al., "Sofosbuvirdaclatasvir-simeprevir plus ribavirin in direct-acting antiviral-experienced patients with hepatitis C," Clinical Infectious Diseases, vol. 64, no. 11, pp. 1615-1618, 2017.

[33] N. Coppola, C. Minichini, M. Starace, C. Sagnelli, and E. Sagnelli, "Clinical impact of the hepatitis $\mathrm{C}$ virus mutations in the era of directly acting antivirals," Journal of Medical Virology, vol. 88, no. 10, pp. 1659-1671, 2016.

[34] E. Lontok, P. Harrington, A. Howe et al., "Hepatitis C virus drug resistance-associated substitutions: state of the art summary," Hepatology, vol. 62, no. 5, pp. 1623-1632, 2015.

[35] E. Lawitz, F. Poordad, J. A. Gutierrez et al., "svr12 results from the phase Ii, open-label Impact study of simeprevir (smv) in combination with daclatasvir ( $\mathrm{dcv}$ ) and sofosbuvir (sof) in treatment-naïve and-experienced patients with chronic $\mathrm{Hcv}$ genotype 1/4 infection and decompensated liver disease: 39 ," Hepatology, vol. 62, p. 227A, 2015.

[36] E. M. Said, B. A. Abdulaziz, M. El Kassas, I. H. El Attar, M. Emadeldeen, and S. M. Abd-Elsalam, "High success rates for the use of sofosbuvir/ombitasvir/paritaprevir/ritonavir+ribavirin and sofosbuvir/simeprevir/daclatasvir + ribavirin in retreatment of chronic hepatitis C infection after unsuccessful sofosbuvir/daclatasvir therapy: a real-life experience," Archives of Virology, vol. 165, no. 7, pp. 1633-1639, 2020.

[37] C. Hézode, S. Chevaliez, G. Scoazec et al., "Retreatment with sofosbuvir and simeprevir of patients with hepatitis $C$ virus genotype 1 or 4 who previously failed a daclatasvir-containing regimen," Hepatology, vol. 63, no. 6, pp. 1809-1816, 2016.

[38] E. Wahsh, A. Hussein, A. Gomaa, M. Baraka, and M. Abead, "Safety and efficacy of combination therapy (simeprevir/ sofosbuvir) in the treatment of chronic HCV genotype IV patients," Medicine Science, vol. 7, no. 1, pp. 122-126, 2018.

[39] M. V. Lacaita, A. Carpentieri, S. Buongiorno et al., "Dermatologic reactions in patients with hepatitis $\mathrm{C}$ receiving direct-acting antiviral (DAAs)," Giornale Italiano di Farmacia, vol. 30, no. 3, pp. 158-163, 2016.

[40] M. Bourliere, S. Gordon, S. Flamm et al., "Sofosbuvir, velpatasvir, and voxilaprevir for previously treated HCV infection," New England Journal of Medicine, vol. 376, no. 22, pp. 2134-2146, 2017.
[41] Clinical Review Report, Sofosbuvir/Velpatasvir/Voxilaprevir (Vosevi), (Gilead Sciences Canada, Inc.), Indication, Hepatitis C infection genotype 1 to 6, Canadian Agency for Drugs and Technologies in Health http://creativecommons.org/licenses/ by-nc-nd/4.0/Bookshelf ID:NBK534028. 